\title{
A Model for Generating Synthetic Dendrites of Cortical Neurons
}

\author{
Jaime Fernández ${ }^{1}$, Laura Fernández ${ }^{1}$, Ruth Benavides-Piccione ${ }^{2,3}$, \\ Inmaculada Ballesteros-Yañez ${ }^{4}$, Javier DeFelipe ${ }^{2,3}$, and José-María Peña ${ }^{1}$ \\ 1 Departamento de Arquitectura y Tecnología de Sistemas Informáticos, \\ Universidad Politécnica de Madrid, Madrid, Spain \\ 2 Laboratorio de Circuitos Corticales (Centro de Tecnología Biomédica), \\ Universidad Politécnica de Madrid, Madrid, Spain \\ ${ }^{3}$ Instituto Cajal, Consejo Superior de Investigaciones Científicas, Madrid, Spain \\ - Facultad de Químicas, Universidad de Castilla-La Mancha, Ciudad Real, Spain
}

\begin{abstract}
One of the main challenges in neuroscience is to define the detailed structural design of the nervous system. This challenge is one of the first steps towards understanding how neural circuits contribute to the functional organization of the nervous system. In the cerebral cortex pyramidal neurons are key elements in brain function as they represent the most abundant cortical neuronal type and the main source of cortical excitatory synapses. Therefore, many researchers are interested in the analysis of the microanatomy of pyramidal cells since it constitutes an excellent tool for better understanding cortical processing of information. Computational models of neuronal networks based on real cortical circuits have become useful tools for studying certain aspects of the functional organization of the neocortex. Neuronal morphologies (morphological models) represent key features in these functional models. For these purposes, synthetic or virtual dendritic trees can be generated through a morphological model of a given neuronal type based on real morphometric parameters obtained from intracellularly-filled single neurons. This paper presents a new method to construct virtual dendrites by means of sampling a branching model that represents the dendritic morphology. This method has been contrasted using complete basal dendrites from 374 layer II/III pyramidal neurons of the mouse neocortex.
\end{abstract}

Keywords: Bioinformatics, Machine Learning, Neurosciencie, Microstructure Neuronal Morphology.

\section{Introduction}

Over the years, neuroscience has evolved considerably thanks to the use of a great variety of in-vivo and in-vitro approaches. However, the study of the nervous system is still subject to several major limitations which comes from three major factors: (i) large number of elements (ii) the existence of an intricate web of interactions between these elements, and (iii) limited knowledge of the functional significance of these interactions. 
To overcome some of these limitations, simulation and modeling mechanisms are proposed to manage the inherent complexity of the nervous system. These mechanisms take profit of the development of new tools integrated with laboratory research using experimental approaches.

In general, neurons adopt a considerable variety of shapes and sizes, as well as different patterns of dendritic and axonal arborizations. In particular, cortical pyramidal neurons consist of a typically pyramid cell body that gives rise to an apical and basal dendritic arbor. Their axons leave the region of origin, in which the cell body is located, and are therefore also called projection neurons.

One of the main challenges in neurascience is to define the detailed structural design of the nervous system. This challenge is one of the first steps towards understanding how neural circuits contribute to the functional organization of the nervous system. In particular, the neocortex is the choice of numerous theoreticians and experimentalists because of its direct involvement in many aspects of mammalian behavior. In the neocortex, pyramidal neurons are key elements in its functional organization as they represent the most abundant cortical neuronal type (70-85\%) and the main source of cortical excitatory synapses. They constitute the vast majority of projection neurons and are commonly subdivided according to their projection site and the pattern of their terminal axonal arborization (for reviews see [1], [2], [3], [4], [5], [6], [7], [8], [9]). Moreover, all dendritic surfaces of pyramidal cells are covered by spines, except the proximal segments arising directly from the cell soma which are spine-free. These dendritic spines constitute the major postsynaptic elements of excitatory synapses. Thus, dendritic spine are considered to be fundamental for memory, learning and cognition $([10],[11],[12],[13])$. There is a spatial segregation of different inputs in different regions of the dendritic tree that can be divided into two major compartments: the apical dendrite with its collateral branches and dendritic tuft, and the basal dendrites. The basal dendrites form about $90 \%$ of the dendritic length of any cortical pyramidal neuron ([14]). Consequently, the basal dendritic arbor represents the major source of synaptic inputs to pyramidal neurons.

As a result, many researchers are interested in the analysis of the microanatomy of pyramidal cells since it constitutes an excellent tool for better understanding cortical processing of information. Computational models of neuronal networks based on real cortical circuits have become useful tools for studying certain aspects of the functional organization of the neocortex $([15])$. A powerful method to examine developmental mechanisms and structure-function relationships of neuronal morphological parameters is computational modeling of neuronal morphology (e.g., [16]). For these purposes, synthetic or virtual dendritic trees can be generated through a morphological model of a given neuronal type based on real morphometric parameters obtained from intracellularly-filled single neurons.

This paper presents a new method to construct virtual dendrites by means of sampling a branching model that represents the dendritic morphology. This method has been contrasted using complete basal dendrites from 374 layer II/III pyramidal neurons of the mouse neocortex. 
The rest of the paper is organized as follows: In Section 2 the main concepts about the morphology of pyramidal cells are briefly presented. Section 3 introduces the methodology to create the morphology model and the use of this model to create virtual dendrites. In Section 4 experimental results are reported. Finally, Section 5 discuses the results and future work.

\section{Dendritic Morphologies of Pyramidal Cells}

A remarkable characteristic of pyramidal cells is the great variations in their microanatomy, since significant differences between pyramidal cells in different cortical layers and areas and between species exist regarding the pattern of dendritic arborisation and in the number and density of dendritic spines (e.g., [14], [17], [18], [19], [20], reviewed in [21], [22]). This is an important issue in terms of function, since the morphology of the dendritic tree is related to the processing of synaptic inputs. For example, the structure of the dendritic tree itself affects the process of integration, while its size influences topographic sampling map and the mixing of inputs ([23],[24], for a recent work see [25]).

In general, a major limitation in analyzing the morphology of the neurons is that it is necessary to use relatively thin tissue slices to visualize labeled neurons, frequently in the order of a few microns, in contrast to the hundreds of microns or even millimeters over which neuronal processes may expand. Thus, labeled processes are frequently incomplete because during the slicing procedures of the tissue some parts of the neuron morphology are missing in a variable degree depending on the thickness of the sections and the relative localization of the labeled neuron within the slice. Currently, this problem can only be overcome by using serial sections to reconstruct the cell in 3D. However, neuronal processes are not always easy to trace and they may be lost in the background noise at times ([26]). Together, these obstacles make it very laborious and timeconsuming to obtain meaningful measurements from neurons. Virtual neurons may also help to validate and develop algorithmic methods for repairing ([27]), using different sampling protocols. The advantage to obtain such a repair method is that pyramidal neurons might be examined in coronal sections to include both the apical and basal dendritic systems, though incomplete the repair methods might help to generate a complete picture of the pyramidal cell morphology.

Fortunately, the basal dendritic arbors of pyramidal cells can be fully reconstructed in single sections. Furthermore these sections are made in the horizontal plane and with a sufficient thickness as to include the whole dendritic tree (e.g., [17]). Thus, we have used data from fully reconstructed basal dendrites as they are particularly valuable to validate and develop methods to create virtual neurons.

\section{Methodological Approach}

Dendrites are complex tree-like 3D structures defined by many morphometric parameters. As a preliminary step towards the creation of virtual dendrites, this 
paper proposes a method to define the branching scheme of the dendrites. Instead of considering the 3D structure of the dendrite, the branching scheme represents some topological aspects, such as the number of branches and the symmetry of these branches, together with some aggregated morphometric parameters, such as the length of the dendrite (and branches) the distance to the cell soma and the tortuosity.

The method presented in this paper has two main components: (i) a procedure to construct a branching model and (ii) a dendrite sampler to use the model to produce virtual dendrites.

The construction of the model is an off-line process applied on the experimental data extracted from real-neurons. How this model is constructed is fully explained in Section 3.2. Nevertheless, it is easier to understand the objective of this model if the dendrite sampler (that uses this model) is first presented.

\subsection{Dendrite Sampler}

The dendrite sampler implements an algorithm to create virtual dendrites by means of sampling a branching model. The virtual dendrites created by this algorithm should be biologically feasible in terms of morphological aspects and statistically analogous to real dendrites obtained by experimental methods.

The dendrite sampler operates at the level of segment. A segment is the portion of a dendrite between two consecutive branch bifurcations, or between the soma and the first bifurcation of the dendrite. A segment has a given pathlength (the length of the corresponding portion of the dendrite, named segment pathlength). The pathlength is also computed between the end of the segment and the cell soma. Another parameter is the euclidean distance between the end of

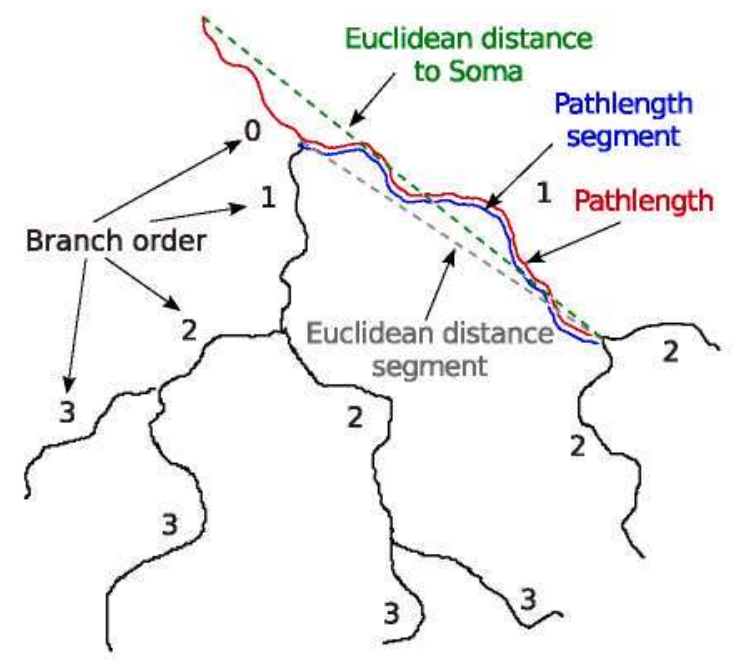

Fig. 1. Dendrite attributes 


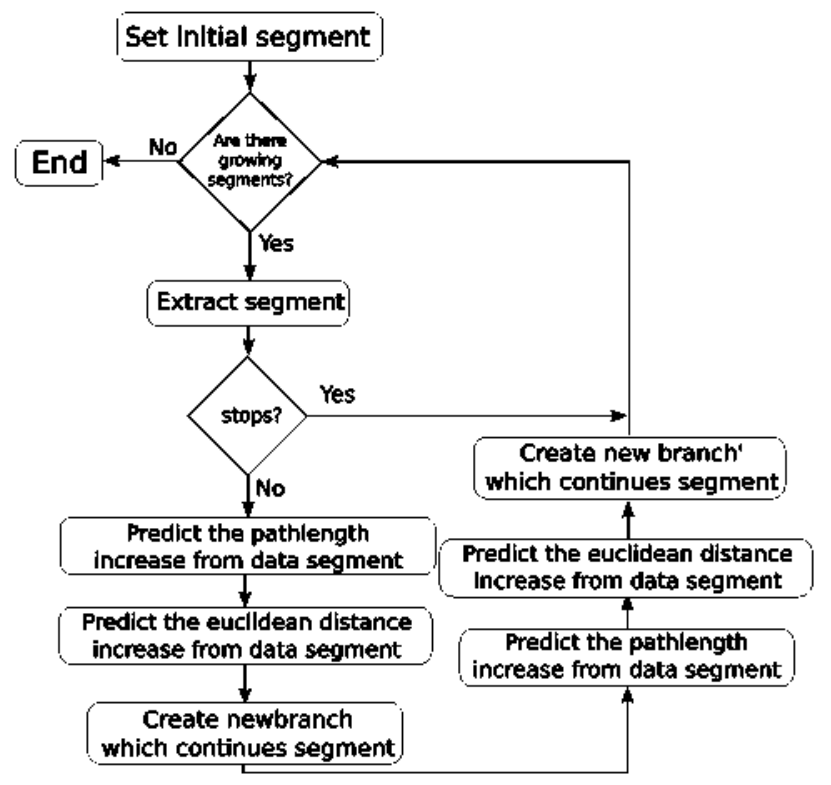

Fig. 2. Sampler flow diagram

the segment and the soma, as well as the euclidean distance between the beginning and the end of the segment. The ratio between this last euclidean distance and the pathlength is called tortuosity. Most of the attributes have been extracted from the literature $([28],[29],[30])$ and there is a graphical explanation in Figure 1.

The dendrite sampler tries to simulate the growth of a real dendrite, it creates the branches in a breadth-first order. In Figure 2 there is a flow diagram to explain the procedure. There is a set of segments, called growing segments, which are in development stage. The sampler extracts the shortest (minimum global pathlength) one and uses the termination model to predict if the branch finishes at this position. If not, the segment has to bifurcate. The bifurcation is performed sampling a pathlength and a euclidean distance for each of the branches (using the pathlength model and the euclidean distance molel). Then, both branches are included in the set of growing segments. The process is repeated iteratively until there are no growing branches in the set.

\subsection{Model Construction}

In the present study we have used homogeneous collections of complete basal dendritic arbors to generate synthetic dendritic morphologies using machine learning methods based in stochastic algorithms. In particular, the whole basal dendritic arbor of layer II/III pyramidal neurons was examined. In this experiment, these cells were obtained from 6 different cytoarquitectonic cortical areas of the mouse 
neocortex: primary and secondary motor (M1 and M2), primary and secondary somatosensory (S1 and S2) and primary and secondary visual cortex (V1 and V2) using intracellular injections of Lucifer Yellow in fixed cortical tissue. Thereafter, the geometrical structure of basal arbors was three-dimensionally reconstructed in each cortical region using Neurolucida package (MicroBrightField). A total of 374 neurons were included in the analysis ( 44 in $\mathrm{M} 1,91$ in $\mathrm{M}^{2}, 44$ in $\mathrm{S} 1,71$ in S2, 41 in V1 and 83 in V2). This material has been used in previous studies ([19], [20] ) in which the intracellular injection methodology and visualization of neurons is described in detail.

In order to obtain a virtual dendrite, the sampler uses three different models: termination model, pathlength model and euclidean distance model. These models are induced from the experimental data provided by the 374 pyramidal cells.

The real dendrite data is retrieved with Neurolucida and stored in ASC format. This format supports basically the morphology structure of the dendrite with space coordinates and additional information like labels, color and other items specified by the Neurolucida user. To use this data to build models and extract the metrics there must be a preprocess of data. It consists of two phases, the first one retrieves the morphology structure of the dendrite, and the second one calculates the different measures of the branches. Once this is done, the information is stored at the segment level. Thus the instances are formed by the data of two consecutive segments, the attributes of the first one, and the finish value, pathlength increment and euclidean distance increment of the second segment. For example, if a segment $A$ bifurcates into two children segments $B$ and $C$, the following two instances will be created $(A, B)$ and $(A, C)$. These instances are used by pathlength model and euclidean distance model. The termination model uses a different dataset containing whole branches (from soma to the end of the branch). This data set records the pathlength and euclidean distance of the branches.

To maintain the quality of the data, there is a filter which excludes from the datasets those dendrites that are too small to exists in a real neuron. These are dendrites with only one branch or one bifurcation. The models are built with Weka from the final dataset and then provided to the sampler for generating the virtual dendrites.

The termination molel uses a normal distribution of the dendrite pathlength estimated from the expermiental data. The sample mean and variance are computed via maximum likelihood (using Bessel correction for the variance). The corresponding model, for each candidate branch, provides a probability to finish at the current pathlength. Then, a binomial distribution is sampled with probability $p$ equals to the probability computed before. This binomial distribution indicates if the branch finishes or not.

The pathlength model is computed by sampling a normal distribution, but in this case the parameters are predicted using a regression model from the data. This regression model approximates the mean value of the increment pathlength using the rest of the information of the previous segment (branch order, previous segment pathlength, previous euclidean distance to soma, ...). The predicted 
value is considered the mean value of the normal distribution, for the variance the standard mean square error (MSE) is used.

The euclidean distance model is created using a very similar process. The main difference is that the model computes the euclidean distance increment. To construct this model the pathlength of the current segment (already sampled) is included.

\section{Experimental Results}

To validate the procedure several morphometric parameters have been used. These measure the whole dendrites, not segments: Total length (pathlength segment of each of the branches of the dendrite), Number of branches (number of branches in the dendritic tree), and Maximum euclidean distance (The euclidean distance reached by the most distant terminal of the branches of the dendrite).

These morphometric parameters have been extensively used in the literature, as they provide important information to construct functional models ([16], [13]).

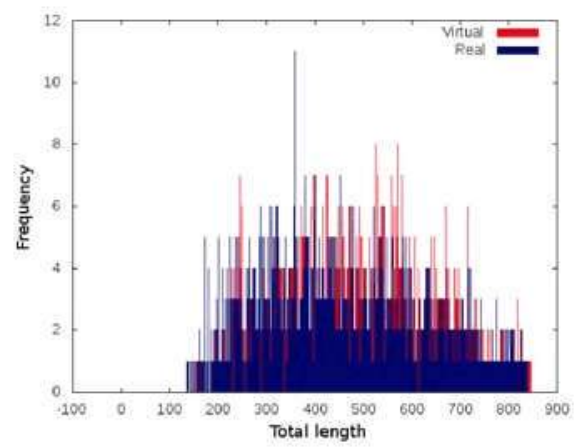

(a) Total length

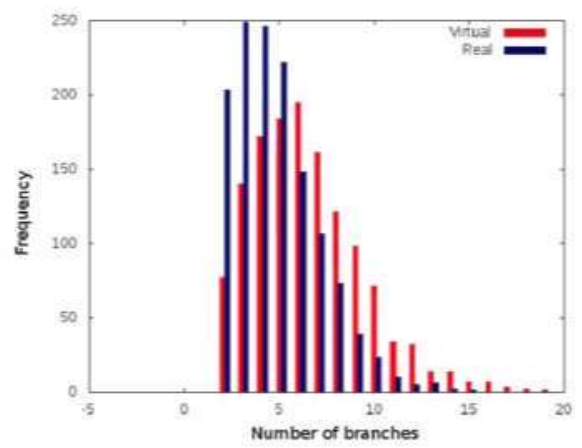

(b) Number of branches

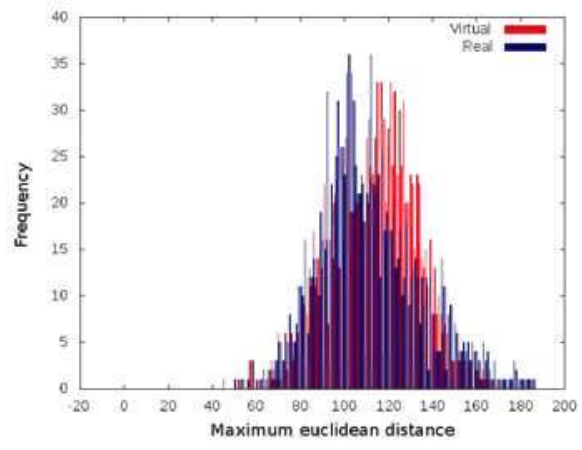

(c) Maximum euclidean distance

Fig. 3. Morphometrics comparison between all the real and virtual dendrites used 
Table 1. Morphometrics p-values of T-Test and F-Test. Bold face indicates distributions with no significant statistical differences.

\begin{tabular}{|c|c|c|c|c|c|c|}
\hline \multirow{2}{*}{ fold } & \multicolumn{2}{|c|}{ Total length } & \multicolumn{2}{c|}{ Max. euclidean distance } & \multicolumn{2}{|c|}{ Number of branches } \\
\cline { 2 - 7 } & T-Test & F-Test & T-Test & F-Test & T-Test & F-Test \\
\hline 0 & 0,41919188 & $\mathbf{0 , 3 6 4 7 8 5 4 7}$ & 0,02830407 & $\mathbf{0 , 1 3 2 4 6 9 9 5}$ & 0,00000289 & 0,00247477 \\
1 & 0,11765158 & $\mathbf{0 , 4 9 7 1 4 1 2 0}$ & 0,05487532 & 0,07755403 & 0,00000022 & 0,00000577 \\
2 & $\mathbf{0 , 6 6 0 6 8 7 7 6}$ & 0,00005603 & $\mathbf{0 , 9 9 4 6 4 9 5 4}$ & 0,01051810 & 0,00000259 & 0,00060837 \\
3 & $\mathbf{0 , 1 2 4 8 1 8 0 6}$ & 0,00132655 & $\mathbf{0 , 4 6 2 2 4 3 3 9}$ & $\mathbf{0 , 1 5 1 3 6 8 7 5}$ & $\mathbf{0 , 0 0 0 0 0 0 3 9}$ & 0,00748735 \\
4 & $\mathbf{0 , 3 3 4 5 8 7 5 9}$ & $\mathbf{0 , 2 9 4 4 9 7 9 1}$ & $\mathbf{0 , 2 1 9 3 1 2 3 1}$ & $\mathbf{0 , 1 5 5 4 4 4 1 3}$ & $\mathbf{0 , 0 0 0 0 0 0 5 5}$ & 0,00465168 \\
5 & $\mathbf{0 , 4 0 1 3 1 1 5 0}$ & $\mathbf{0 , 4 5 8 1 3 7 7 0}$ & $\mathbf{0 , 6 1 3 1 9 3 6 4}$ & $\mathbf{0 , 2 2 5 9 1 7 1 2}$ & $\mathbf{0 , 0 0 0 0 0 1 8 8}$ & 0,00002223 \\
6 & $\mathbf{0 , 9 4 2 5 1 5 1 4}$ & $\mathbf{0 , 3 8 9 6 9 3 6 0}$ & $\mathbf{0 , 0 4 5 1 3 9 7 1}$ & $\mathbf{0 , 6 6 3 9 9 8 3 5}$ & $\mathbf{0 , 0 0 0 1 0 5 0 5}$ & 0,00121797 \\
7 & $\mathbf{0 , 0 0 0 2 6 7 7 3}$ & $\mathbf{0 , 5 1 4 0 1 7 4 8}$ & 0,00011822 & $\mathbf{0 , 4 9 4 5 6 6 6 1}$ & 0,00000000 & 0,00035040 \\
8 & $\mathbf{0 , 0 2 4 8 9 3 6 0}$ & $\mathbf{0 , 8 8 8 0 7 1 4 1}$ & $\mathbf{0 , 0 2 4 0 8 6 9 3}$ & $\mathbf{0 , 3 6 7 5 8 7 0 0}$ & $\mathbf{0 , 0 0 0 0 0 0 2 6}$ & 0,00133224 \\
9 & $\mathbf{0 , 0 0 0 0 6 9 4 9}$ & $\mathbf{0 , 1 3 2 8 0 7 1 8}$ & $\mathbf{0 , 0 0 4 8 8 9 0 2}$ & $\mathbf{0 , 4 5 0 0 6 1 3 2}$ & $\mathbf{0 , 0 0 0 0 0 0 0 0}$ & 0,00000558 \\
\hline
\end{tabular}

The length and number of branches provide information of the complexity of the input maps of the neuron and the euclidean distance an estimation of the interaction radius between neurons. Figure 3 shown a comparison of all this morphometrics values obtained in the process between real and virtual dendrites.

The evaluation of the quality in these models consists on a ten-fold validation. The 374 neurons are randomly separated into 10 different groups (folds). Then, 9 out of these 10 folds are grouped together to create the input data to construct the models. After that, the models are passed through the dendrite sampler and it will produce the same number of dendrites that contains the remaining fold. Then the three morphometric measures are considered to compare the set of real dendrites and the set of virtual dendrites generated by the sampler.

The mechanisms carried out to compare the different measures and determine if there exist statistical differences are two statistical tests: T-Test and the $\mathbf{F}$ Test, which compare the mean and standard deviation distributions, respectively.

The entire process is repeated 10 times, considering each time a different fold for validation and using the rest of them to construct the models. The p-values obtained are shown in Table 1, where the bold face values represent folds which do not have statistical differences between the real and virtual distributions and, therefore, we cannot assert than they are different from each other.

\section{Conclusions}

In this paper, a first approach on the prediction of the morphologies of pyramidal cells has been presented. This study has considered 374 neurons and some morphometric parameters (such as total length of branches, maximum euclidean distance and the number of branches). In order to evaluate the results a methodology of validation of the sampled data has been presented.

This approach shows that even preliminary studies on the prediction of neuronal morphologies can obtain promising good results for some of the 
morphometric parameters. The total pathlength for a given dendrite branch is easily predicted by the distribution of pathlengths in a homogeneous data set.

As the prediction for the total pathlength is well-adjusted the other two models have to compensate each other. The pathlength of the segment is not so precise and probably longer than expected, which reduces the number of branches required to reach the expected pathlength of the entire branch.

In the near future, more effort will be addressed to refine the segment pathlength model to improve the overall sampling procedure, by additionally analyzing the cells grouping them per cortical areas. As an open issue, the model will provide $3 \mathrm{D}$ structures of dendrites and will include other morphological elements.

The expectation of using this model in functional studies of cortical circuits is also clear. Besides the application to construct computational models, these virtual neurons have a short-term practical application to develop repair algorithm methods in order to reconstruct the missing portions of the labeled neurons ([27]). Such a repair method would allow to generate a complete picture of the pyramidal cell morphology in coronal sections, in which both basal and apical arbors are partially included within the sections.

\section{Acknowledgements}

The authors would like to thank the Cajal Blue Brain Project and the Blue Brain Project (EPFL), the technical expertise and assistance provided by CeSViMa and the Spanish Supercomputing Network. This work was supported by the Spanish Ministry of Science under TIN2007 - 67148, SAF2009 - 09394 grants and the Cajal Blue Brain Project.

\section{References}

1. Jones, E.: Anatomy of cerebral cortex: columnar input-output organization. Cerebral Cortex, 199-235 (1981)

2. Jones, E.: The columnar basis of cortical circuitry. In: Willis, W.D. (ed.) The clinical neurosciences, pp. 357-383 (1983)

3. Jones, E.: Laminar distribution of cortical efferent cells. Cerebral Cortex 1, 524-553 (1984)

4. Feldman, M.: Morphology of the neocortical pyramidal neuron. Cerebral cortex, $123-200(1984)$

5. Lund, J.: Anatomical organization of macaque monkey striate visual cortex. Ann. Rev. Neurosci. 11, 253-258 (1988)

6. White, E.L.: Cortical Circuits: Synaptic Organization of the Cerebral Cortex. In: Structure, Function and Theory. Birkhauser, Boston (1989)

7. Felleman, D., Van Essen, D.: Distributed hierarchical processing in the primate cerebral cortex. Cereb. Cortex 1, 1-47 (1991)

8. DeFelipe, J., Farinas, I.: The pyramidal neuron of the cerebral cortex: morphological and chemical characteristics of the synaptic inputs. Prog. Neurobiol. 39, $563-607(1992)$

9. Lund, J., Yoshioka, T., Levitt, J.: Substrates for interlaminar connections in area V1 of the macaque monkey cerebral cortex. Cerebral cortex 10, 37-60 (1994) 
10. Elston, G.: Cortex, cognition and the cell: new insights into the pyramidal neuron and prefrontal function. Cereb. Cortex 13, 1124-1138 (2003)

11. Yuste, R., Bonhoeffer, T.: Genesis of dendritic spines: insights from ultrastructural and imaging studies. Nat. Rev. Neurosci. 5, 24-34 (2004)

12. Lamprecht, R., LeDoux, J.: Structural plasticity and memory. Nat. Rev. Neurosci. 5, 45-54 (2004)

13. Spruston, N.: Pyramidal neurons: dendritic structure and synaptic integration. Nat. Rev. Neurosci. 9, 206-221 (2008)

14. Larkman, A.: Dendritic morphology of pyramidal neurones of the visual cortex of the rat: I. Branching patterns. J. Comp. Neurol., 307-319 (1991)

15. Markram, H.: The blue brain project. Nat. Rev. Neurosci. 7, 153-160 (2006)

16. Donohue, D., Ascoli, G.: A comparative computer simulation of dendritic morphology. PLoS Comput. Biol. 4(5), e1000089 (2008)

17. Elston, G., Rosa, M.: The occipito-parietal pathway of the macaque monkey: comparison of pyramidal cell morphology in layer III of functionally related cortical visual areas. Cereb. Cortex 7, 432-452 (1997)

18. Jacobs, B., Schall, M., Prather, M., Kapler, E., Driscoll, L., Baca, S., Jacobs, J., Ford, I., Wainwright, M., Treml, M.: Regional dendritic and spine variation in human cerebral cortex: a quantitative golgi study. Cereb. Cortex 11, 558-571 (2001)

19. Benavides-Piccione, R., Hamzei-Sichani, F., Ballesteros-Yañez, I., DeFelipe, J., Yuste, R.: Dendritic size of pyramidal neurons differs among mouse cortical regions. Cerebral Cortex 16, 990-1001 (2006)

20. Ballesteros-Yañez, I., Benavides-Piccione, R., Bourgeois, J., Changeux, J., DeFelipe, J.: Topological reorganization of dendritic arbors of cortical pyramidal neurons in mice lacking high-affinity nicotine receptors (submitted 2010)

21. Jacobs, B., Scheibel, A.: Regional dendritic variation in primate cortical pyramidal cells. In: Schz, A., Miller, R. (eds.) Cortical areas: unity and diversity, pp. 111-131. Oxford University Press, Oxford (2002)

22. Elston, G.: Specializations in pyramidal cell structure during primate evolution. Evolution of nervous systems (2007)

23. Koch, $\mathrm{C}_{*}$, Segev, I.: The role of single neurons in information processing. Nat. Neurosci. 3, 1171-1177 (2000)

24. Poirazi, P., Mel, B.: Impact of active dendrites and structural plasticity on the memory capacity of neural tissue. Neuron 29, 779-796 (2001)

25. Wen, Q., Stepanyants, A., Elston, G., Grosberg, A., Chklovskii, D.: Maximization of the connectivity repertoire as a statistical principle governing the shapes of dendritic arbors. Proc. Natl. Acad. Sci. 106, 12536-12541 (2009)

26. DeFelipe, J.: The neuroanatomist's dream, the problems and solutions, and the ultimate aim. Front Neurosci. 2, 10-12 (2008)

27. Anwar, H., Riachi, I., Hilland, S., Schurmann, F., Markam, H.: An approach to capturing neuron morphological diversity. Computational modeling methods for neuroscientists (2009)

28. Donolue, D.E., Ascoli, G.: A comparative computer simulation of dendritic morphology. PLos Computational Biology 4, e1000089 (2008)

29. Uylings, H.B.M., Pelt, J.V.: Measures for quantifying dendritic arborizations. Brain Research 13, 397-414 (2002)

30. Van Pelt, J., Schierwagen, A.: Morphological analysis and modeling of neuronal dendrites. Mathematical Biosciences 188, 147-155 (2004) 